\title{
Association of Lithium Bromide in Acetone
}

\author{
A N N - MARGRET NILSSON, GÖRAN WIKANDER \\ and PER BERONIUS
}

Division of Physical Chemistry, University of Umea, S-901 87 Umea, Sweden

\begin{abstract}
The conductance of lithium bromide in anhydrous acetone at $25^{\circ}$ has been measured over a concentration range of $0.99 \times 10^{-5}$ to $2.6 \times 10^{-8} \mathrm{M}$. The data have been analyzed by means of the Shedlovsky equation for several values of the ion-size parameter, $\dot{a}$, in the Debye. Hückel equation. The "best fit" of this equation to the experimental points was obtained for $\dot{a}=\mathbf{5} .5 \AA$. The ion-pair association constant and the limiting equivalent conductance are compared with data according to other authors.
\end{abstract}

Conductance data for lithium bromide in acetone at $25^{\circ}$ have been previously Ureported by Dippy, Jenkins, and Page, ${ }^{1}$ Savedoff, ${ }^{2}$ Pistoia, Polcaro, and Schiavo, ${ }^{3}$ and by Singh and Mishra. ${ }^{4}$ To determine ${ }^{5}$ the association constant, $K_{\mathrm{A}}$, of this salt in the actual medium we employed the data of these authors. A considerable variation in the calculated values of $K_{\mathrm{A}}$ was, however, observed (Table 2). Further conductance measurements have therefore been undertaken. The parameters derived from the data thereby obtained are compared in the present paper with values calculated from the earlier investigations.

\section{EXPERIMENTAL}

Acetone (Merck, pro analysi) was purified according to Ref. 6 and fractionally distilled in a Vigreux column. The middle cut (specific conductivity $1.4 \times 10^{-8}-2.5 \times 10^{-8} \Omega^{-1} \mathrm{~cm}^{-1}$; water content $5 \times 10^{-3} \%$ by weight as determined by NMR) was immediately used. Lithium bromide (Fluka, suprapur) was dried at $200^{\circ}$ for $2 \mathrm{~h}$. Conductivity water (specific conductivity $0.5 \times 10^{-6}-1.0 \times 10^{-6} \Omega^{-1} \mathrm{~cm}^{-1}$ ) for preparation of potassium chloride solutions was obtained from a quartz distilling apparatus. Potassium chloride (Merck) was of suprapur grade.

A stock $0.01 \mathrm{M}$ lithium bromide solution was prepared by weight. Less concentrated solutions were prepared from this stock by the weight dilution method. All weights were corrected to vacuo. Solutions were protected from the moisture of the air during transfer operations.

A Leeds and Northrup 4666 conductivity bridge, in connection with a Hewlett Packard 201 C audio frequency oscillator and a General Radio Corp. 1232 A amplifier and null detector, was used to measure the resistances of the solutions.

Conductivity cells (Fig. 1), of $30 \mathrm{ml}$ capacity, fitted with bright platinum electrodes, were used. The latter, connected to platinum wires ( $\varnothing 0.5 \mathrm{~mm}$ ) sealed in glass tubes, wer

Acta Chem. Scand. 24 (1970) No. 4 
rigidly held by glass struts. Connection between cell and conductivity bridge was provided by shielded copper wires, each with a resistance of less than $1 \times 10^{-3} \Omega$. The leads were spaced well away from the solution in the cell to avoid the Parker effect.

Four different cells with constants of the order 0.22 and $0.07 \mathrm{~cm}^{-1}$ were used. Calibration was performed according to Lind, Zwolenik, and Fuoss ${ }^{7}$ using potassium chloride solutions of different concentrations in the range $1 \times 10^{-8}$ to $9 \times 10^{-8} \mathrm{M}$. Each cell constant, being an average of several calibrations, was determined with an uncertainty of less than $\pm 0.04 \%$ on the $99.9 \%$ confidence level.

Transfer of test solution to conductivity cell was carried out in a "glove box" containing dry nitrogen under slight overpressure. The cell was rinsed four times with the solution under investigation, filled and placed in a kerosene filled constant temperature bath at

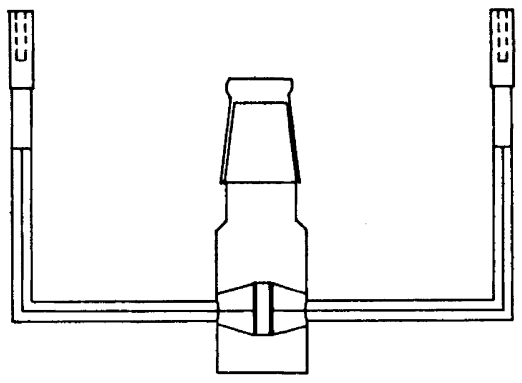

Fig. 1. Schematic picture of conductivity cell.

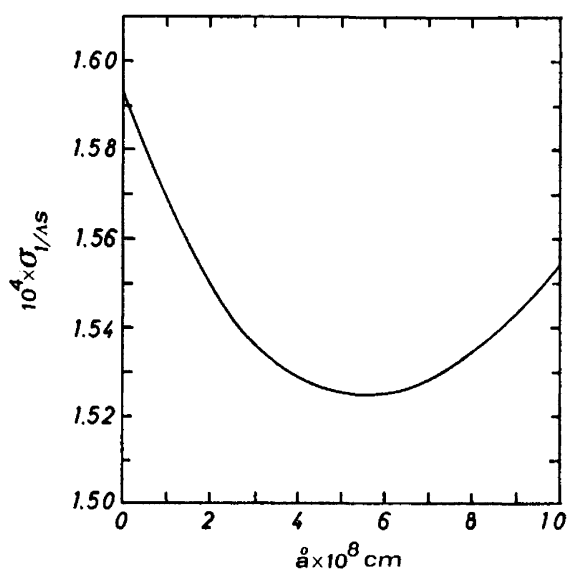

Fig. 2. Standard deviation of the single $1 / \Lambda S$-value as a function of the ion-size parameter, $\dot{a}$.

$25.00 \pm 0.01^{\circ}$ and allowed to equilibrate for $30 \mathrm{~min}$ before measuring the resistance. This procedure was repeated three times for each concentration and the average, corrected for the conductivity of the solvent, used in calculating the equivalent conductance. For each sample, resistances, $r$, were measured at different frequencies, $v$, between 2 and $5 \mathrm{kHz}$ and the resistance extrapolated to infinite frequency; $r$ was plotted against $1 / \nu$ and the curve, being generally of very good linearity, extrapolated to $1 / \nu=0$.

\section{CALCULATIONS AND RESULTS}

Equivalent conductances, $\Lambda$, for several lithium bromide concentrations, c, are quoted in Table 1. The association constant, $K_{\mathrm{A}}$, and the limiting equivalent conductance, $\Lambda_{0}$, were iteratively evaluated by a least squares treatment as previously described ${ }^{8}$ using the Shedlovsky equation ${ }^{9,10}$

$$
\frac{1}{\Lambda S}=\frac{1}{\Lambda_{0}}+\frac{c \Lambda S \gamma^{2}}{K \Lambda_{0}^{2}}
$$

where $S$ is a function of $c, \Lambda, \Lambda_{0}$, solvent properties and temperature, $K=1 / K_{\mathrm{A}}$, and $\gamma$ is the mean molar activity coefficient. The Debye-Hückel equation ${ }^{11}$ 
Table 1. Conductance data for lithium bromide in anhydrous acetone at $25^{\circ}$.

\begin{tabular}{cccc}
\hline $\begin{array}{c}c \times 10^{4} \\
M\end{array}$ & $\begin{array}{c}\Lambda \\
\mathrm{cm}^{2} \Omega^{-1} \text { equiv. }\end{array}$ & $\begin{array}{c}c \times 10^{4} \\
\mathrm{M}\end{array}$ & $\begin{array}{c}\Lambda \\
\mathrm{cm}^{2} \Omega^{-1} \text { equiv. }^{-1}\end{array}$ \\
\hline 26.039 & 54.18 & 2.057 & 126.96 \\
25.794 & 55.41 & 2.007 & 127.11 \\
24.636 & 56.28 & 1.261 & 142.49 \\
21.215 & 58.63 & 1.077 & 146.16 \\
19.258 & 61.94 & 1.066 & 142.93 \\
19.148 & 61.95 & 0.9961 & 148.49 \\
10.727 & 75.17 & 0.9938 & 153.49 \\
10.631 & 77.05 & 0.7535 & 163.72 \\
9.707 & 79.55 & 0.5548 & 163.86 \\
9.647 & 79.48 & 0.5060 & 176.15 \\
9.610 & 78.67 & 0.2789 & 175.06 \\
4.845 & 97.81 & 0.2498 & 186.27 \\
4.010 & 105.86 & 0.1075 & 190.34 \\
4.006 & 105.42 & 0.09909 & \\
2.272 & 121.15 & &
\end{tabular}

was used to evaluate the last. The values 20.7 and $3.16 \times 10^{-3}$ poise for the dielectric constant and viscosity of the solvent were used.

In calculating $K_{\mathrm{A}}$ and $\Lambda_{0}$ the question arose how to choose the ion-size parameter, $\dot{a}$, in calculating activity coefficients. Several values were tried to investigate which value would give the "best fit" of eqn. (1) to the data in Table 1 . The results of these calculations, performed with the aid of a CDC 3200 computer, are shown graphically in Fig. 2, where the standard deviation in the single $1 / \Lambda S$-value, calculated in the usual manner, ${ }^{12}$ is given as a func-

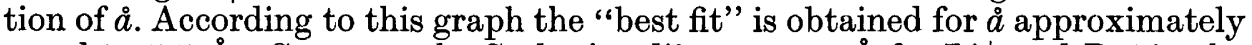
equal to 5.5 $\AA$. Compare the Stokes' radii sum $5.48 \AA$ for $\mathrm{Li}^{+}$and $\mathrm{Br}^{-}$in the actual solvent according to Savedoff, ${ }^{2}$ and the crystal radii ${ }^{13}$ sum $2.55 \AA$.

Values of $K_{\mathrm{A}}$ and $\Lambda_{0}$ for the latter values of $\dot{a}$ are given in Table 2 in which errors quoted are standard deviations. For comparison the corresponding

Table 2. Association constants and limiting equivalent conductances according to conductance data of various investigators.

\begin{tabular}{|c|c|c|c|c|}
\hline \multirow{3}{*}{ Ref. } & \multicolumn{2}{|c|}{$\dot{a}=2.55 \AA$} & \multicolumn{2}{|c|}{$\dot{a}=5.48 \AA$} \\
\hline & $K_{\mathrm{A}}$ & $\Lambda_{0}$ & $K_{\mathrm{A}}$ & $\Lambda_{0}$ \\
\hline & $\mathrm{M}^{-1}$ & $\mathrm{~cm}^{2} \Omega^{-1}$ equiv..$^{-1}$ & $\mathbf{M}^{-1}$ & $\mathrm{~cm}^{2} \Omega^{-1}$ equiv. ${ }^{-1}$ \\
\hline 1 & $2229 \pm 37$ & $164.1 \pm 2.2$ & $2111 \pm 29$ & $162.6 \pm 1.7$ \\
\hline 2 & $4467 \pm 79$ & $193.7 \pm 2.7$ & $4375 \pm 79$ & $193.1 \pm 2.7$ \\
\hline 3 & $3910 \pm 11$ & $196.8 \pm 0.8$ & $3643 \pm 10$ & $192.9 \pm 0.8$ \\
\hline 4 & $605 \pm 16$ & $135.5 \pm 1.3$ & $557 \pm 14$ & $134.1 \pm 1.1$ \\
\hline This work & $4676 \pm 34$ & $198.9 \pm 1.7$ & $4520 \pm 33$ & $197.7 \pm 1.7$ \\
\hline
\end{tabular}

Acta Chem. Scand. 24 (1970) No. 4 
values according to previous conductance data, ${ }^{1-4}$ re-evaluated by the Shedlovsky method as outlined above, have been included. An attempt to fit the latter data ${ }^{1-4}$ to the Fuoss-Onsager equation ${ }^{14}$ was not successful in all cases. The Shedlovsky method was therefore used throughout to enable a direct comparison of all results.

Table 2 reveals that the association constant and limiting equivalent conductance according to the present investigation agree fairly well with the values derived from conductance data of Savedoff ${ }^{2}$ and from data of Pistoia, Polcaro, and Schiavo. ${ }^{3}$

Values of $K_{\mathrm{A}}$ and $\Lambda_{0}$ according to Dippy, Jenkins, and Page ${ }^{1}$ and according to Singh and Mishra are considerably lower. A possible reason for these low values might be the presence of polar impurities in the solvent media used. Dippy, Jenkins, and Page ${ }^{1}$ treated their acetone with calcium chloride and potassium carbonate to remove water. This treatment may, however, result in formation of diacetone alcohol. ${ }^{15,16}$ Singh and Mishra ${ }^{4}$ do not specify their method of purification, but in view of the high conductivity $\left(0.3 \times 10^{-6}\right.$ to $0.5 \times 10^{-6} \Omega^{-1} \mathrm{~cm}^{-1}$ ) of the acetone used by these investigators, the solvent has not been adequately purified.

Acknowledgements. The authors thank Dr. Michael Sharp for linguistic revision of the manuscript and the Swedish Natural Science Research Council for financial support.

\section{REFERENCES}

1. Dippy, J. F. J., Jenkins, H. O. and Page, J. E. J. Chem. Soc. 19391386.

2. Savedoff, L. G. J. Am. Chem. Soc. 88 (1966) 664.

3. Pistoia, G., Polcaro, A. M. and Schiavo, S. Ric. Sci. 37 (1967) 227.

4. Singh, D. and Mishra, A. Bull. Chem. Soc. Japan 40 (1967) 2801.

5. Beronius, P., Isacsson, U. and Nilsson, A.-M. Acta Chem. Scand. 24 (1970) 189.

6. Smith, S. G., Fainberg, A. H. and Winstein, S. J. Am. Chem. Soc. 83 (1961) 618.

7. Lind, J. E., Zwolenik, J. J. and Fuoss, R. M. J. Am. Chem. Soc. 81 (1959) 1557.

8. Beronius, P. Acta Chem. Scand. 23 (1969) 1175.

9. Shedlovsky, T. J. Franklin Inst. 225 (1938) 739.

10. Fuoss, R. M. and Shedlovsky, T. J. Am. Chem. Soc. 71 (1949) 1496.

11. Robinson, R. A. and Stokes, R. H. Electrolyte Solutions, Butterworths, London 1959, p. 229.

12. Youden, W. J. Statistical Methods for Chemists, Wiley, New York 1951, Chap. 5.

13. Pauling, L. The Nature of the Chemical Bond, Cornell University Press, New York, N.Y. 1940, Chap. X.

14. Fuoss, R. M. and Onsager, L. J. Phys. Chem. 61 (1957) 668.

15. Timmermans, J. and Gillo, L. Roczniki Chem. 18 (1938) 812.

16. Weissberger, A., Ed., Technique of Organic Chemistry, Interscience, Inc., New York 1955, Vol. VII, p. 380 .

Received October 18, 1969. 